\title{
SMD Detection and Classification Using YOLO Network Based on Robust Data Preprocessing and Augmentation Techniques
}

\author{
NDAYISHIMIYE Fabrice ${ }^{1}$, Joon Jae Lee ${ }^{2 *}$
}

\begin{abstract}
The process of inspecting SMDs on the PCB boards improves the product quality, performance and reduces frequent issues in this field. However, undesirable scenarios such as assembly failure and device breakdown can occur sometime during the assembly process and result in costly losses and time-consuming. The detection of these components with a model based on deep learning may be effective to reduce some errors during the inspection in the manufacturing process. In this paper, YOLO models were used due to their high speed and good accuracy in classification and target detection. A SMD detection and classification method using YOLO networks based on robust data preprocessing and augmentation techniques to deal with various types of variation such as illumination and geometric changes is proposed. For 9 different components of data provided from a PCB manufacturer company, the experiment results show that YOLOv4 is better with fast detection and classification than YOLOv3.
\end{abstract}

Key Words: PCB inspection, SMD inspection, Classification, Detection, YOLO.

\section{INTRODUCTION}

Surface mount technology (SMT) [1] as shown in Fig. 1, is a method in which electronic components are mounted onto the printed circuit board (PCB) [2] to reduce manufacturing costs and make more efficient use of the PCB space. Surface mount devices (SMD) [3] are electronic components used within the surface mount assembly process and it is possible recently to build highly complex electronic circuits into smaller and smaller assemblies. While these days almost everything is shifting to an online system due to the pandemic situation, PCB manufacturing is becoming more and more important due to the strong demand for the consumer of electronics products like computers, tablets, smartphones which are more useful in our daily life. More fields such as automobile, medical industries, aeronautics need also these electronic devices. Therefore, as the demands of these electronics are increasing so fast, the production of the PCB should also be fast as well to satisfy the demands. Nowadays, Automatic optical inspections (AOI) [4] are widely used in manufacturing, assembling the PCB for detecting the errors and ensuring the quality of the final product. The most basic of the AOI inspection items is to check whether a specific part is installed in the correct position. Hence, a learning mode is required, which is a process of registering the specifications of the SMD to be mounted in a specific location in advance before inspection as a reference sample or model. The analysis of components mounted on the PCB is very time-consuming as most inspections rely on manual labor. In addition, since a person manually checks the detailed name of the component, a problem may occur due to an operator's misjudgment or mistake which conduct to low performance. Therefore, the detection of these components based on a convolutional neural network (CNN) [5] is the key operation for PCB

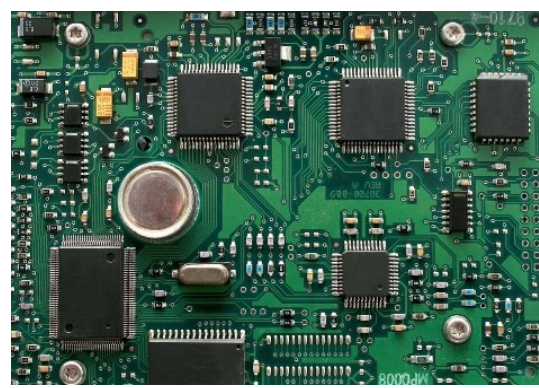

Fig. 1. Printed circuit board with electronic components.

\footnotetext{
Manuscript received October 14, 2021; Revised November 21, 2021; Accepted November 30, 2021. (ID No. JMIS-21M-09-034) Corresponding Author (*): Joon Jae Lee, Keimyung University, Daegu, Republic of Korea, +82 53580 6682, joonlee@kmu.ac.kr ${ }^{1}$ Department of Computer Engineering, Keimyung University, Daegu 42601, Republic of Korea, ndayifab2@gmail.com, joonlee@kmu.ac.kr
} 
and manufacturing companies to reach the quality of their products. When a new board is introduced, the algorithm inspects the component by classifying the types of components at a specific location later in the inspection process and comparing whether the component is identical or not to the component registered in the design process. The machine can then classify a PCB component as good, no good or defective based on a scratch, a small hole, the presence of nanoparticles such as dust, paper fragments, small air bubbles or component shift compared to the reference sample.

This paper proposes a PCB electronic components detection and classification method using YOLO (You Look Only Once) algorithms. YOLO [6] is a kind of fast object detection method based on a convolutional neural network. The deep network architecture of CNN can detect discrimination features from all the input images, so we do not need experts to define image features. To verify the effectiveness of the proposed approach, a dataset of real PCB component images was collected and trained by different versions of YOLO but for company privacy reasons, this dataset is not open to the public. Major components mounted on a PCB are generally resistors, capacitors, inductors, potentiometers, transformers, diodes, transistors, integrated circuits, oscillators, switches, and sensors. But we mainly focus as explained in detail in section 3, on the detection of 9 different PCB components includes C-CHIP, R-CHIP, DIODE, TRANSISTOR, LCHIP, CAPACITOR, IC, COIL and LED by using YOLOv3 [7] and v4 [8], the effective and accurate object detectors. These algorithms are becoming increasingly popular because of their best accuracy, speed, and efficiency. The image is checked just once as the name itself defines YOLO. A single forward propagation pass is performed by the neural network to generate predictions. Following the non-max suppression (NMS) [9] technique, the algorithm outputs the recognized objects using the bounding box.

The main contributions proposed in this paper are summarized as follows. First, images are collected and preprocessed to have robust classification characteristics against various colors and shapes of the component and slight rotation changes. Second, to improve the classification performance, we generate some new data by applying brightness based on mean and standard deviation of the original images and by generating customized anchor boxes. As result, both detectors based on YOLO v3 and v4 show good performance with an average of $99 \%$ in the experiments.

The rest of the paper is as follows. In section 2, we review some works related to this study. Section 3 describes the methodology applied in this study for PCB components classification and target recognition. Section 4 shows the implementation and the results of our experiments and finally, this study ends with a conclusion and future work in section 5 .

\section{RELATED WORK}

\subsection{Automatic Optical Inspection and Component Classification}

An Automatic optical inspection is an important tool that aids in detecting faulty components on a PCB. This technique utilizes high-performance scalable modular camera technology with four color illuminations from all spatial directions to produce optimum contrast value for many defects during feature extractions. There are three kinds of AOI methods include image comparison [10], key point comparison [11] and feature comparison [12]. The image comparison method mainly detects defects by pixel difference between standard image and test image. This kind of detection method is simple to use and fast in detection, but it requires high accuracy of image location and matching. Because of the variety of plug-in polar capacitors, non-uniform, high size, large position offset, the simple image comparison method has a high false detection rate and poor stability. The key point comparison method is used to detect errors by locating and analyzing the key points of components.

The classification method of these nine components can be divided into large, medium, and small classification as defined in [13] where the authors focus on middle classification. The criterion for dividing into the middle class is the size of the component based on the width and length. In the case of large classification, it is further subdivided and classified using the depth of the component as well as the type, width and length. The middle classification of devices is width $\times$ length according to each type, and the small classification is divided into width $\times$ length $\times$ type $\times$ depth. In the case of small classification, the width and length of components are almost the same, so it can be distinguished only by depth information. That is, RGBD 4-channel image information is required. Even though the authors in [13] used the medium classification, the PCB manufacturer suggested using the large classification to compare the results with the medium classification and to analyze the devices without taking into consideration of sizes and then for further study, focus on classifying the small component as soon as we collect data with depth information.

Before classifying the middle classification components, the class name of the component depends on the width and length of the component as shown in Table 1, 
Table 1. Collected data and class name by type.

\begin{tabular}{|c|c|c|c|c|c|}
\hline Large & Medium & Class name & Large & Medium & Class name \\
\hline \multirow{7}{*}{$\begin{array}{l}\text { Capacitor } \\
\text { (chip) }\end{array}$} & C-CHIP_0402Size & 0402-C & \multirow{6}{*}{ Resistor } & R-CHIP_0603Size & $0603-\mathrm{R}$ \\
\hline & C-CHIP_0603Size & 0603-C & & R-CHIP_1005Size & $1005-\mathrm{R}$ \\
\hline & C-CHIP_1005Size & $1005-\mathrm{C}$ & & R-CHIP_1608Size & $1608-\mathrm{R}$ \\
\hline & C-CHIP_1608Size & $1608-\mathrm{C}$ & & R-CHIP_2012Size & 2012-R \\
\hline & C-CHIP_2012Size & $2012-\mathrm{C}$ & & R-CHIP_3216Size & $3216-\mathrm{R}$ \\
\hline & C-CHIP_3216Size & $3216-\mathrm{C}$ & & R-CHIP_3225Size & $3225-\mathrm{R}$ \\
\hline & C-CHIP_3225Size & $3225-\mathrm{C}$ & \multirow{4}{*}{ Transistor } & TR_3PinTR_1816Size & 1816-T \\
\hline \multirow{4}{*}{ Diode } & DIODE_0603Size & 0603-D & & TR_3PinTR_2012Size & $2012-\mathrm{T}$ \\
\hline & DIODE_10065Size & 10065-D & & TR_3PinTR_2812Size & $2812-\mathrm{T}$ \\
\hline & DIODE_1511Size & 1511-D & & TR_6PinTR_2812Size & 2812-T-6 \\
\hline & DIODE_2515Size & $2515-\mathrm{D}$ & \multirow[b]{2}{*}{ LED } & LED_big_size & LED_big \\
\hline Capacitor & Capacitor & Capacitor & & LED_small_size & LED_small \\
\hline \multirow{4}{*}{ COIL } & COIL_C_2219Size & COIL_C_2219 & \multirow{5}{*}{ IC } & IC_2_vertical & IC_2_vertical \\
\hline & COIL_C_4140Size & COIL_C_4140 & & IC_2_horizontal & IC_2_horizontal \\
\hline & COIL_S_3023Size & COIL_S_3023 & & IC_4_0 & IC_4_0 \\
\hline & COIL_S_3220Size & COIL_S_3220 & & IC_4_1 & IC_4_1 \\
\hline L-CHIP & L-0402Size & L-0402 & & IC_4_2 & IC_4_2 \\
\hline \multicolumn{2}{|l|}{ Large class } & 9 classes & & Medium class & 34 classes \\
\hline
\end{tabular}

and the type of the component as well. The color of the board may be different from one PCB manufacturer to another but most of these manufacturers use the standardized shape and size width of $10 \mu \mathrm{m}$ (micrometer) and a length of $5 \mu \mathrm{m}$ (micrometer), it is class 1005-C. The first two numbers of class are the horizontal length, the last two numbers are the vertical length, and the last alphabet is the first letter of SMD. If the component is s chip capacitor and has the component type. This is how the class names are determined and the details are shown in Table 1.

\subsection{PCB Defect Detection using Deep Learning}

Robust and precise defect detection is a great significance in the production of high-quality PCB. Due to the complexity of $\mathrm{PCB}$ production environments, most previous works still utilize traditional image processing and matching algorithms to detect PCB defects. In [14], an improved bare PCB defect detection approach is proposed by learning deep discriminative features, which also greatly reduced the high requirement of a defect dataset with some artificial defect and affine transformation to increase the quantity and diversity of defect data. Then, a deep pretrained convolutional neural network is employed to learn high-level discriminative features of defects. They finetune the base model on the extended dataset by freezing all the convolutional layers and training the top layers. Finally, the sliding windows approach is adopted to further localize the defects. Extensive comparison with three additional shallow feature-based methods demonstrates that the proposed approach is more feasible and effective in the PCB defect detection area. [15] also presented a deep learning neural network and hybrid genetic algorithm for PCB defect detection. Deep learning neural network was used for classification. For feature selection, a genetic algorithm was applied to optimize the feature reduction process.

In [16] the authors talk about some challenges in the field of PCB detection due to the various component's shape and size. Therefore, there are some more challenges to solve and collaboration needed from the hardware assurance and security community for automated, accurate, and scalable PCB component detection.

Image processing is a part of computer vision [17] that performs some operations on an image to get an enhanced image or to extract some useful information from it. The main operations are segmentation, classification, and detection. The idea behind object detection is to find the region of interest (ROI) [18] in the image, get the category information and location information of these regions or objects. However, these objects appear in different shapes and positions with the interference of various factors like occlusion, lighting, and many other factors. Hence, object detection has many challenges in the field of computer vision.

Object detection is a computer vision task that involves predicting the presence of one or more objects, along with their classes and bounding boxes (location information). YOLO uses the features of the entire image to predict the bounding box and classify or detect the targets within the 
box, indicating that the YOLO network can use the full information existing in the image to achieve target classification and position detection. Yolo algorithm is generalized to outperform various strategies between natural and various fields from object detection. The purpose of the algorithm is to classify objects using CNN. This algorithm is much easier and simpler to use in realtime than other classifier algorithms. YOLOv3 and v4 produce better detection results based on speed, accuracy, FPS, mAP than SSD [19]. YOLO is a state-of-the-art object detector that can perform object detection in real-time with good accuracy. YOLOv4 is one of the popular algorithms used in object detection due to its tremendous speed and capability of detecting objects in real-time with good accuracy. The first three YOLO versions which are YOLOv1 released in 2016, YOLOv2 in 2017 and YOLOv3 in 2018 also showed some good results but YOLOv4 is better in speed and accuracy than the previous versions. YOLOv3 and v4 are not only faster in detection but are also more suitable for small target detection. The only difference between YOLOv4 from yolov3 is based on the backbone. The YOLOv3 has a Darknet53 backbone while the YOLOv4 has CSPDarknet53. Other things are quietly the same compared with YOLOv3. The YOLOv4 heads are the same as YOLOv3. The Head is the prediction part, and it has two types. One is dense prediction (One-stage detector) and another one is sparse prediction (Two-stage detector). The Prediction module can make predictions by using the feature extracted from the network. Taking a $13 \times 13$ grid, for example, is equivalent to dividing the input picture into $13 \times 13$ grids, and then each grid will be preset with three prior frames. The prediction results of the network will adjust the positions of the three prior frames, and finally, it will be filtered by the non-maximum suppression algorithm to obtain the final prediction frame.

\section{METHODOLOGY}

\subsection{Type of SMDs}

SMD is usually smaller, and it has either smaller leads or sometimes no leads at all and it may have short pins or leads of various styles, flats contacts. Therefore, the inspection is a very important step to ensure product performance along with the miniaturization of semiconductor components and the increase in PCB microprocessing. The most basic of the inspection items is to check whether a specific part is installed in the correct position. For this, a learning model is required, which is a process of registering the specifications of the SMD to be mounted in a specific location in advance before the inspection.

SMDs consist of electrical elements such as diodes, capacitors, resistors, and more. For a PCB to function effectively, each component must play its role. If one part fails, then the PCB may fail to function as intended. In this study, as the main purpose is to classify these components, we selected 9 different components as 9 classes.

Capacitors are often categorized according to the conductor or dielectric material, which gives rise to many types, and we only consider two of them which are chip capacitors as C-CHIP and the classic capacitors with two leads as CAPACITOR. We also consider chip resistors as R-CHIP, L-CHIP to indicate an inductor chip, COIL as part of transformers, Light-emitting-diode as LED, Integrated Circuit as IC, TRANSISTOR, and DIODE as shown in Fig. 2, the samples of all 9 classes covered in this study.

The component image used in registration and inspection is not an image of the entire PCB as shown in Fig. 2 but the image of the part to be inspected is cut out separately from the PCB image. A $700 \times 700$-pixel image is extracted from the entire $\mathrm{PCB}$ with one component to be inspected located on the center of the image. Components mounted on the PCB are mounted horizontally or vertically depending on the PCB design. In this study, the component with the least lead is rotated upward to change the shape of the component during the image processing step. The reason for using the $700 \times 700$ images is because the resolution is good enough to extract features and there is not a single component larger than this resolution. Using this resolution, it is easy to center the component and extract useful information from it and more practical to know where the components will be mounted on the PCB based on the completed design at the design stage.

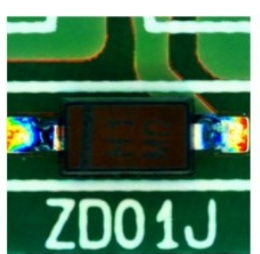

DIODE

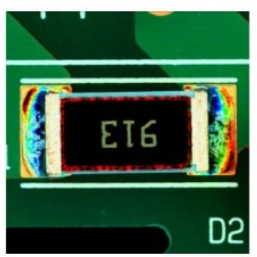

R-CHIP

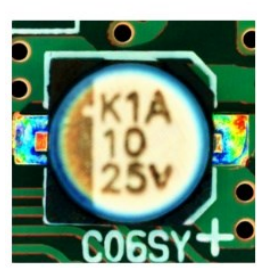

CAPACITOR

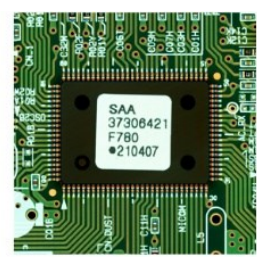

IC

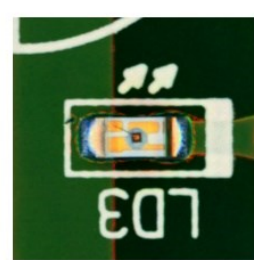

LED

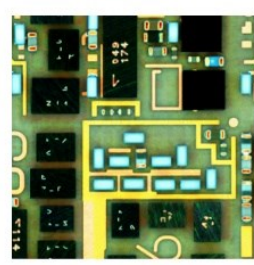

L-CHIP

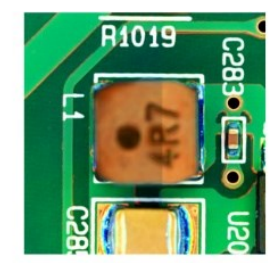

COIL

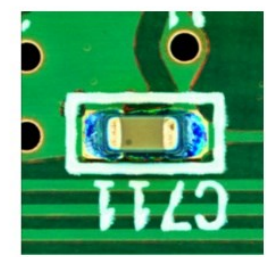

C-CHIP

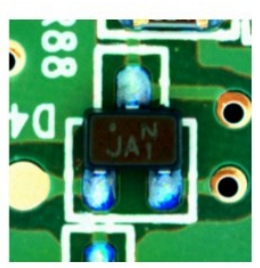

TRANSISTOR
Fig.2. Data sample for each class. 


\subsection{Image Pre-processing and Data Augmentation}

\subsubsection{Illumination variation}

To implement a robust model for detection and classification of SMD found on PCB based on deep learning and referred to some techniques used in [20] and [21] for data sampling, variety in the dataset is more important for a suitable model for different kinds of PCB manufacturers. Therefore, image pre-processing is applied to enhance the original image so that the result is more convenient for subsequent operations and applications than the original image. There are several used methods such as image rotation, linear transformation, histogram transformation, gamma transformation, brightness and contrast adjustment. For brightness and contrast adjustment, increasing or decreasing $\beta$, which control the brightness, will add or subtract a constant value for each pixel. $\beta$ may improve the image brightness, but at the same time, the contrast may be reduced. Therefore, $\alpha$ will be used to reduce this effect. We also need to control the value of brightness $\beta$ because if we add more brightness the image may lose some details of the original bright area and cause the saturation.

Generally, to adjust the brightness and contrast, they use the multiplication and an addition with a constant as shown in the next equation.

$$
g(x)=\alpha f(x)+\beta,
$$

where the parameter $\alpha>0$ and $\beta$ control the contrast and brightness respectively. $f(x)$ is the input image pixel, $g(x)$ is the output image pixel and $x$ is a color component value $\mathrm{R}, \mathrm{G}$, and $\mathrm{B}$.

In this study, the value of $\alpha$ depends on the initial image mean $\mu 1$ which is the image pixel intensity and the target image mean $\mu 2$.

$$
\alpha=1+\frac{\beta}{255} .
$$

Here $\beta$ is the mean difference between the initial image and the target image.

As shown in Fig. 3, this is the representation of C-CHIP class training data distribution in grayscale based on mean and standard deviation where the data distribution is not enough because there are some missed data in the circle which can be an issue as we need to make a robust deep learning model for PCB component classification and detection.

Therefore, after applying the method explained above by increasing the image brightness and contrast, we generate some new images as shown on the circle in the circle. However, the mean intensity below 30 is not useful because the image is too dark while the mean intensity above 200 is too bright with some color saturation.

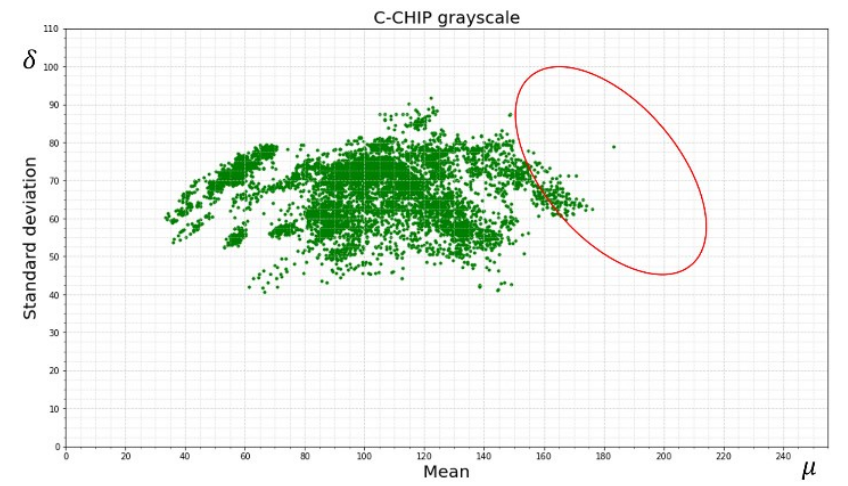

Fig. 3. C-CHIP grayscale original collected data distribution.

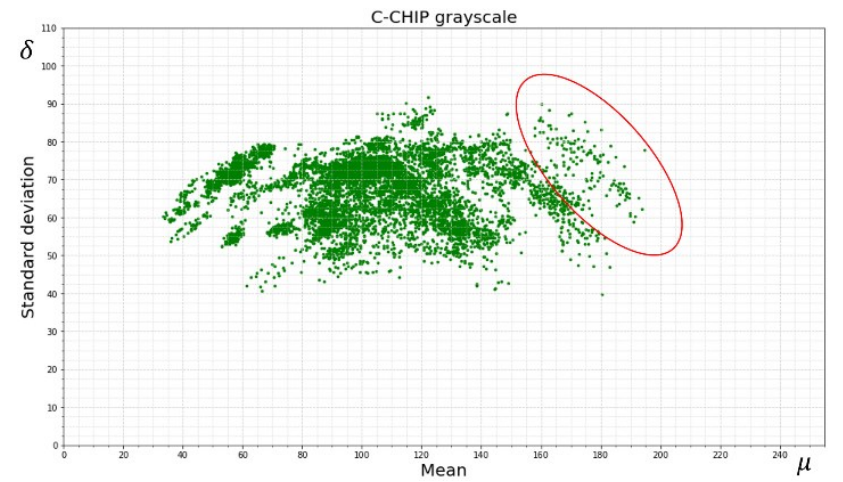

Fig. 4. The result of data distribution for C-CHIP grayscale after applying some illumination variation.

The result of this illumination variation as shown in Fig. 5, the preprocessed image is brighter than the input image and the data distribution is wider than before.
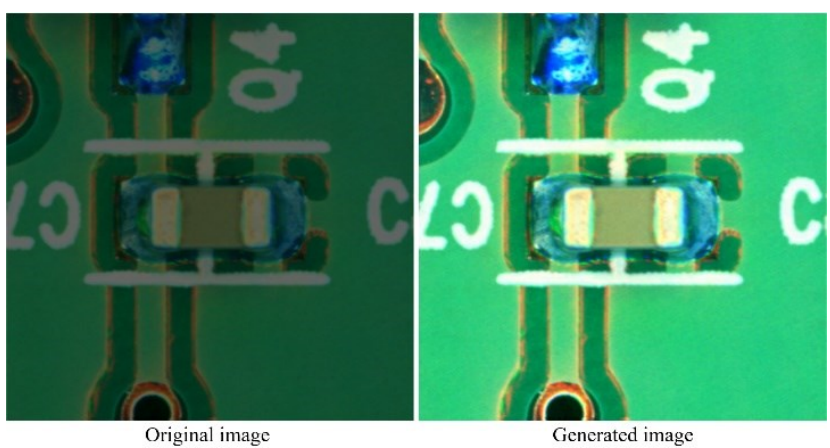

Fig. 5. Generated data brighter than the original data.

\subsubsection{Scale variation and image rotation}

As the aim of this study is focused on the classification and detection of components, collecting training data taken at different scales is more helpful for the classifier to learn the same component at different scales.

Many important ideas in geometry can be understood using transformations. As explained in the previous section, the devices are small in size (micrometer) and during the device printing, the rotation of the device to a very small angle result in uncertain camera calibration. 


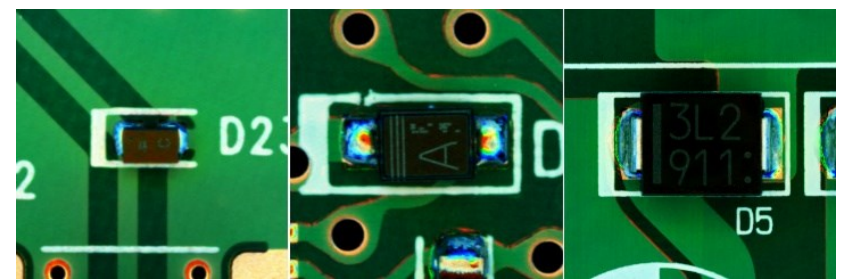

Fig. 6. Diode with similar shape and color but different size, 100065-D(left), 1511-D(middle) and 4235-D(right).

Here are geometric transformations consisting of rotations about the original image. Let us consider a rotation of the original image through a slight angle $\theta$. The angle is a fixed angle between -5 to +5 degrees same as some cases in the real PCB components. Any point (x, y) in the plane rotates at a distance from the origin based on the angle $\theta$ with the positive x-axis, as illustrated in Fig. 7 below.

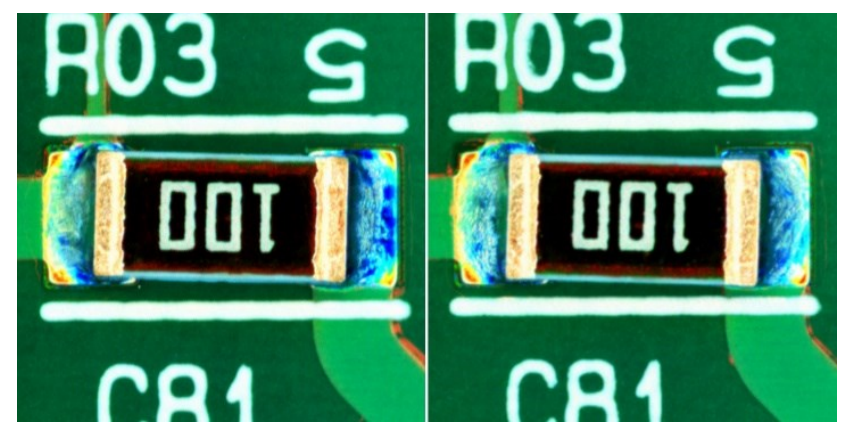

Fig. 7. Resistor component with a slight rotation.

\subsubsection{Geometric variation}

In collected data, they are some data in the same class with different shapes and sizes as shown in Fig. 8, these are all IC components, but the left component has many small leads on both two sides while the IC on right has many leads on four sides of the component. This is one of some features which differentiate the IC from other components. As we focus on the large classification to make a robust model for SMD detection, the model will learn the component in their different form and shapes.

\subsubsection{Data labeling}

In this paper, the method of computer vision detection is used to classify the various PCB components which are very important to train the model with an image containing information about PCB components. However, these components appear in different types according to their size and shape. The dataset used in this study is obtained from a PCB assembly company.

Component annotation is completed using a graphical image annotation tool and label object bounding boxes in images. The annotation files are stored in XML format with the image name, component location, component type.
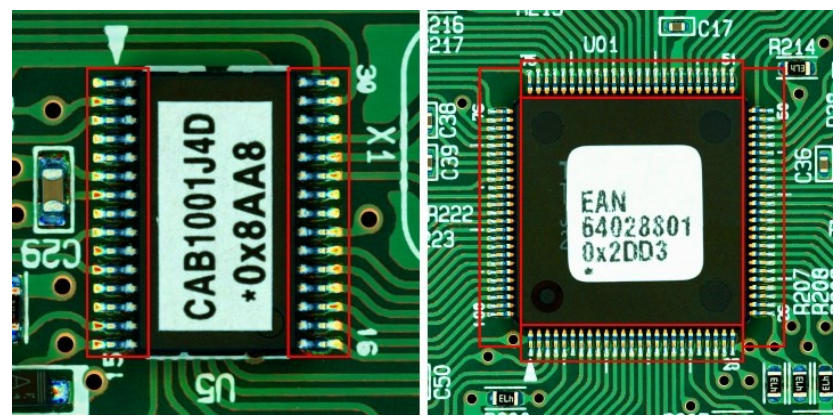

Fig. 8. Integrated Circuit (IC) with different geometric variation, IC with leads in both sides of the component on left and IC with leads on 4 sides of the component.
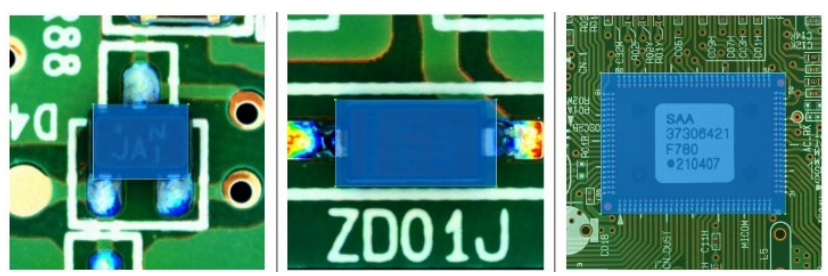

Fig. 9. Training data labeling. Transistor on the left, Diode in the middle and IC on the right.

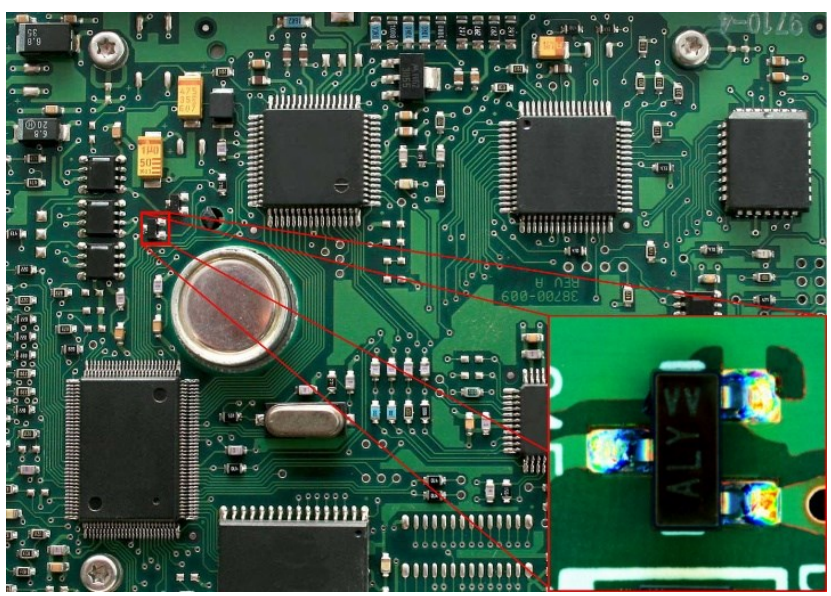

Fig. 10. Extracted image from a PCB image for training.

The component location is the pixel coordinates of the target object as shown in Fig. 9 to localize the component's bounding box, finally, the component type is the type that belongs to all 9 classes taken into consideration in this study. Each component has its characteristics that facilitate the model during the feature extraction on the backbone stage. Therefore, during the labeling, we also include the component lead as shown in Fig. 9 where DIODE has two leads, TRANSISTOR has three leads and IC has many leads.

YOLOv3 has the advantages of detection speed and accuracy and meets the real-time requirements for object detection. However, YOLOv3 has many backbone network parameters and requires high hardware performance, which is not conducive to the popularization of applications because it consumes a lot of memory as shown in the results 
of our experiments. On the other hand, YOLOv4 is an important improvement of YOLOv3 by the modification of the architecture in the Backbone and the Neck based on BoF (bag of freebies) and BoS (bag of specials), the Path aggregation network (PAN) [22] and the Cross-iteration batch normalization (CBN) [23]. The BoF improves the accuracy of the detector without increasing the inference time while BoS improve the accuracy of object detection. Hence, the YOLO v4 became more efficient and suitable for single GPU training and fust during inference with good accuracy.

\subsubsection{Anchor boxes}

Object detection models utilize anchor boxes to make bounding box predictions. Understanding and carefully tuning the model with customized anchor boxes can be a very important step to improve the object detection model's performance, especially when the objects are in different shapes. The anchor's significance is that its size predefines the target's most likely length and width to be detected. In the data preprocessing of the YOLOv3, we usually use Kmeans to cluster the target sizes in the training set to generate nine most likely target anchors, each with its width and height. The size of the images in the dataset is often not uniform, and all images, whether for training or test in the YOLOv3 or YOLOv4, need to be resized to $608 \times 608$ first. Therefore, the anchors are generated after resizing the training set image in advance. The advantage of this is that all the data are resized in advance to meet the size of the network input, and the width or length of the anchor can be directly used as the threshold of the effective receptive field size in the three anchors distribution layers. After calculation of the PCB train dataset, the sizes of the 9 anchors generated after normalizing are $(39,20)$; $(72,37)$; (125, 69); (193, 102); (234, 158); (303, 146); (381, 219); $(286,327) ;(457,421)$.

\section{EXPERIMENT AND RESULTS}

We implement YOLO v3 and v4 algorithms to classify our custom $\mathrm{PCB}$ dataset classified into 9 different classes include C-CHIP, R-CHIP, DIODE, TRANSISTOR, LCHIP, CAPACITOR, IC, COIL and LED which are electronic components found on a PCB board. These algorithms had shown good performance on different stateof-the-art datasets like MS coco, Pascal VOC, and others more. The purpose is to provide a fast detection system of large classes without consideration of the component sizes. We resized all the original images from $700 \times 700$ pixels to $608 \times 608$ pixels and all experiments were performed in the environment as shown in Table 2.
Table 2. Computing environment.

\begin{tabular}{|l|l|}
\hline Device & Configuration \\
\hline Operation System & Windows 10 \\
\hline Memory & 32GB RAM \\
\hline \multirow{2}{*}{ Processor } & Intel Core i7-6700K CPU \\
& $@ 4.00 \mathrm{GHz}$ \\
\hline GPU accelerator & CUDA 10.2, cuDNN 8.0.2 \\
\hline \multirow{2}{*}{ GPU } & NVIDIA 2x GeForce GTX \\
& 1080 \\
\hline
\end{tabular}

We trained these models with the same amount of training dataset, same test set and the same 9 anchor boxes to check which model is more suitable for SMD detection and classification. For each class in the training set, we select at least 1000 images to build a robust model for electronic components based on different sizes and shapes. Per class, $80 \%$ of the data were for training data and $20 \%$ for the validation set.

Table 3. Experimental data both for YOLOv3 and v4.

\begin{tabular}{|c|c|c|c|}
\hline Num & Class & Training set & Test set \\
\hline \hline 1 & C-CHIP & 8202 & 2900 \\
\hline 2 & R-CHIP & 6909 & 1610 \\
\hline 3 & TRANSISTOR & 3194 & 7491 \\
\hline 4 & COIL & 1662 & 410 \\
\hline 5 & DIODE & 3443 & 1130 \\
\hline 6 & LED & 1401 & 1599 \\
\hline 7 & CAPACITOR & 1028 & 1126 \\
\hline 8 & L-CHIP & 1000 & 173 \\
\hline 9 & IC & 3407 & 3178 \\
\hline
\end{tabular}

During the inference, we tested the model with enough new data also based on different sizes and shapes to verify how good are these models in the detection and classification of PCB components. We refer to the real situation where we may find a lot of test data.

We compare the model predict class with the ground-truth class. As shown in Table 4, If the predicted class and the ground-truth class are the same, the model classifies well the component which is pass. On the other hand, if the predicted component is different from the ground-truth component, the model fails to make a good classification of the component.

The results of these two algorithms applied on SMDs are interesting because the detectors are fast, accurate with fast detection and classification average time. However, there are some problems of misclassification and detection failure for C-CHIP and R-CHIP. This is the case where the model confuses the C-CHIP component to an R-CHIP and vice-versa, also there are some components that the model could not recognize at all. 
SMD Detection and Classification Using YOLO Network Based on Robust Data Preprocessing and Augmentation Techniques

Table 4. The experiment results with YOLOv3 and YOLOv4.

\begin{tabular}{|c|c|c|c|c|c|c|c|c|c|c|}
\hline \multirow{2}{*}{ Class } & \multirow{2}{*}{ Count } & \multicolumn{3}{|c|}{ YOLOv3 } & \multicolumn{3}{c|}{ YOLOv4 } \\
(with data augmentation) & \multicolumn{3}{c|}{ YOLOv4 } \\
(without data augmentation) \\
\cline { 3 - 12 } & & Pass & Fail & Accuracy & Pass & Fail & Accuracy & Pass & Fail & Accuracy \\
\hline \hline C-CHIP & 2900 & 2873 & 27 & $99.06 \%$ & 2898 & 2 & $99.93 \%$ & 2898 & 4 & $99.86 \%$ \\
\hline R-CHIP & 1610 & 1606 & 4 & $99.75 \%$ & 1610 & 0 & $100 \%$ & 1610 & 0 & $100 \%$ \\
\hline TRANSISTOR & 7491 & 7491 & 0 & $100 \%$ & 7491 & 0 & $100 \%$ & 7491 & 0 & $100 \%$ \\
\hline COIL & 410 & 410 & 0 & $100 \%$ & 410 & 0 & $100 \%$ & 410 & 0 & $100 \%$ \\
\hline DIODE & 1130 & 1125 & 5 & $99.55 \%$ & 1130 & 0 & $100 \%$ & 1106 & 24 & $97.87 \%$ \\
\hline LED & 1599 & 1599 & 0 & $100 \%$ & 1599 & 0 & $100 \%$ & 1599 & 0 & $100 \%$ \\
\hline CAPACITOR & 1126 & 1126 & 0 & $100 \%$ & 1126 & 0 & $100 \%$ & 1126 & 0 & $100 \%$ \\
\hline L-CHIP & 173 & 173 & 0 & $100 \%$ & 173 & 0 & $100 \%$ & 173 & 0 & $100 \%$ \\
\hline IC & 3178 & 3178 & 0 & $100 \%$ & 3178 & 0 & $100 \%$ & 3178 & 0 & $100 \%$ \\
\hline \multicolumn{2}{|c|}{ TOTAL } & 19581 & 36 & $99.81 \%$ & 19615 & 2 & $99.99 \%$ & 19589 & 28 & $99.85 \%$ \\
\hline
\end{tabular}

In YOLOv4, the use of BoF and BoS make the object detector accurate by increasing the training cost but by improving the inference cost.

To verify the importance of applying the data augmentation proposed in this research, we add an experiment with original data without applying the data augmentation on YOLOv4 as shown in Table 4. The experiment with data augmentation shows good results compared to the experiment on original data without data augmentation.

Table 5. The YOLOv4 model evaluation.

\begin{tabular}{|c|c|c|c|c|c|}
\hline Model & $\mathrm{mAP}_{50}$ & $\mathrm{mAP}_{75}$ & Precision & Recall & F1-score \\
\hline YOLOv4 & $99.23 \%$ & $99.16 \%$ & 0.84 & 0.99 & 0.91 \\
\hline
\end{tabular}

Table 5 shows the model evaluation where it achieves an mAP of $99.23 \%$ by using an intercession-over-union (IoU) threshold of $50 \%$ and an mAP of $99.16 \%$ for a threshold of $75 \%$.

Fig. 11 shows the two fail components in the YOLOv4 experiment result. Our prediction is to detect the component located in the center of the image. But the image on left, the detector detects two devices which are C-CHIP and Diode, and both are close to the center of the image, but none is in the center and the Diode probability is higher than C-CHIP. On the other hand, the image on right detects the component as an R-CHIP while it is a C-CHIP. However as shown in Fig. 12, this C-CHIP has some similarities with R-CHIP like the black part inside the component which is one of the main features of the C-CHIP device.

\section{CONCLUSION}

In this paper, to classify and detect SMD on the PCB board, the characteristics of each component are considered, the boundary box of the components is generated based on our target object, and classes are defined. And most important we used some data preprocessing and augmentation methods to build a robust model which detects and classifies the SMDs during the inspection step. As we used our customized data, the anchor boxes were also modified. To build a robust deep learning model we used the large classification so that each class will have various data shapes and sizes. We customized the YOLO algorithms based on our dataset of electronic SMDs for detection and classification tasks. The effectiveness and robustness of these models are shown in the previous section. But based on results, YOLOv4 always shows the best results compared to the other models. YOLOv4 detector is very accurate and effective for SMD detection and classification with an mAP of $99.16 \%$ for a threshold of $75 \%$. However, there are still errors or misclassified and undetected data in our models like some components that failed to be classified and detected.

Based on these limitations in this study, we will extend our analysis by collecting more data with depth information and making a small classification taking into account the size and shapes of the device. 


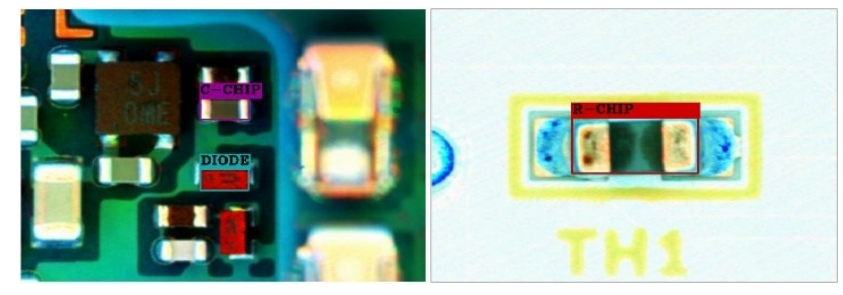

Fig. 11. The 2 C-CHIP fails data in the YOLOv4 experiment result.

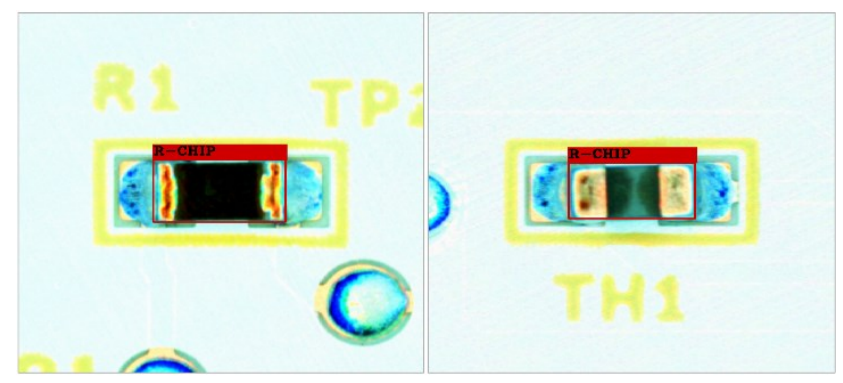

Fig. 12. Comparison of similarity between R-CHIP component and C-CHIP component.

\section{Acknowledgment}

This research was supported by Basic Science Research Program through the National Research Foundation of Korea (NRF) funded by the Ministry of Education (NRF2020R1I1A3074667).

\section{REFERENCES}

[1] Hui, T., Pang, G., "Solder paste inspection using region-based defect detection," The International J. of Advanced Manufacturing Technology, vol. 42, pp. 725-734, 2009.

[2] Ananthu Raj, and A. Sajeena, "Defects Detection in PCB Using Image Processing for Industrial Applications," in Proceedings of the Second International Conference on Inventive Communication and Computational Technologies (ICICCT), Sept. 2018.

[3] Soukup, R., "A methodology for optimization of false call rate in automated optical inspection post reflow," in Proceedings of the 33rd International Spring Seminar on Electronics Technology, pp. 263-267, Aug. 2010.

[4] F Wu, X Zhang, Y Kuan, Z He, “An AOI algorithm for PCB based on feature extraction," in Proceedings of the 7th World Congress on Intelligent Control and Automation, Aug. 2008.

[5] M. D. Zeiler and R. Fergus, "Visualizing and understanding convolutional networks," in Proceedings of the Europian Conference on Comput. Visi. ECCV, pp. 818-833, 2014.
[6] J. Redmon, S. Divvala, R. Girshick, and A. Farhadi, "You only look once: Unified, real-time object detection," in Proceedings of the IEEE Conf. Comput. Vis. Pattern Recognit, pp. 779-788, 2016.

[7] Redmon, Joseph, and Ali Farhadi, "Yolov3: An incremental improvement," arXiv preprint arXiv, 1804.02767, 2018.

[8] Bochkovskiy Alexey, Chien-Yao Wang, and HongYuan Mark Liao, "YOLOv4: Optimal Speed and Accuracy of Object Detection." arXiv preprint arXiv, 2004.10934, 2020.

[9] A. Mrutyunjay, P. Kondrakunta, H. Rallapalli, "Nonmax Suppression for Real-Time Human Localization in Long Wavelength Infrared Region," Advances in Decision Sciences, Image Processing, Security and Computer Vision, vol. 4, pp. 166-174, 2019.

[10] F. Ardhy, F.I. Hariadi, "Development of SBC based Machine-Vision System for PCB Board Assembly Automatic Optical Inspection," in Proceedings of International Symposium on Electronics and Smart Devices (ISESD), 2016.

[11] P. Resa, I. Farkhad, "Development Of Techniques to Determine Object Shifts for PCB Board Assembly Automatic Optical Inspection (AOI)," in Proceedings of International Symposium on Electronics and Smart Devices (ISESD), 2018.

[12] X. Hongwei, K. Yongcong, Z. Xianmin, “A High Speed AOI Algorithm for Chip Component Based on Image Difference," in Proceedings of IEEE International Conference on Information and Automation, pp. 969-974, 2009.

[13] H. Yoon, J. Lee, "PCB Component Classification Algorithm Based on YOLO Network for PCB Inspection," Journal of Korea Multimedia Society, vol. 24, pp. 998-999, 2021.

[14] X. He, S. Zhang, S. Liu, S. Yang, "Automatic Crimping of PCB Electrical Connectors for Satellite Electronic Products," Electronics Process Technology, vol. 2, pp. 1-5, 2016.

[15] P. K. Srimani, Pratibha, "Adaptive Data Mining Approach for PCB Defect Detection and Classification," Indian Journal of Science and Technology, vol. 9, no. 44, pp. 1-9, 2016.

[16] M. A. Mallaiyan Sathiaseelan, O. P. S.Paradis, Taheri, N. Asadizanjani, "Why Is Deep Learning Challenging for Printed Circuit Board (PCB) Component Recognition and How Can We Address It?," Cryptography, vol. 5, no. 1, pp. 1-9, 2021.

[17] W. Zhao, R. Chellappa, J. Phillips, and A. Rosenfeld, "Face recognition: A literature survey," $A C M$ Computing Surveys, vol. 35, pp. 399-458, 2003.

[18] Q. Wang, L. Yau, Z. Xiaoke, H. James, "Region of 
interest selection for functional features," Neurocomputing, vol. 422, pp. 235-244, 2021.

[19] Wei Liu, Dragomir Anguelov, Dumitru Erhan, Christian S., Scott R., Cheng-Yang F., Alexander C. Berg, "SSD: Single shot multibox detector," in Proceedings of the Europian Conference on Computer Vision - ECCV, pp. 21-37, 2016.

[20] Gang, S., Fabrice, N., Chung, D., Lee, J., "Character Recognition of Components Mounted on Printed Circuit Board Using Deep Learning," Sensors, vol. 21, 2021.

[21] S. Gang, J. Lee, "Coreset Construction for Character Recognition of PCB Components Based on Deep Learning," Journal of Korea Multimedia Society, vol. 24, pp. 382-395, 2021.

[22] L. Shu, L. Qi, Q. Haifang, S. Jianping, J. Jiaya, "Path Aggregation Network for Instance Segmentation," in Proceedings of the IEEE Conference on Computer Vision and Pattern Recognition (CVPR), pp. 87598768, 2018.

[23] Z. Yao, Y. Cao, S. Zheng, G. Huang, S. Lin, "CrossIteration Batch Normalization," in Proceedings of the IEEE/CVF Conference on Computer Vision and Pattern Recognition (CVPR), pp. 12331-12340, 2021.

Authors

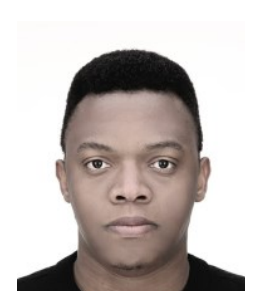

NDAYISHIMIYE Fabrice received his bachelor's degree from Hope Africa University, Burundi in 2016. In 2020, he received his MS degree at Keimyung University, Korea and now he is pursuing his Ph.D. degree in the Computer Engineering department. His research interests include deep learning, image processing, pattern recognition.

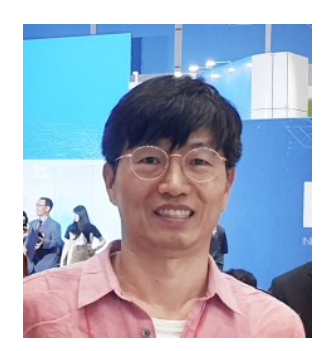

Joon Jae Lee received his BS, MS, a Ph.D. degree from Kyungpook National University, Korea in 1986, 1990, and 1994, respectively. From 1995 to 2007, he was an associate professor at Dongseo Univ. He also was a visiting professor at Georgia Institute of Technology from 1998 to 1999 . He worked for Parmi corporation as a director from 2000 to 2001. In September 2007, he joined the Faculty of Computer Engineering at Keimyung University, Korea, where he is currently a professor. His research interests include computer vision, image processing, pattern recognition, and deep learning. 